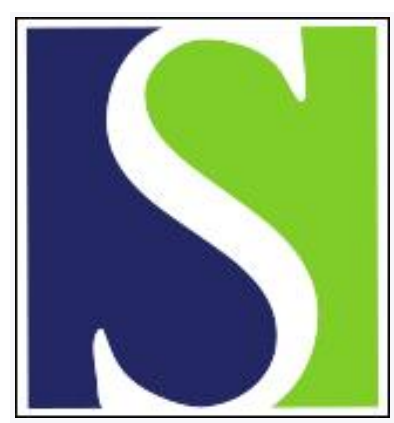

Scand J Work Environ Health 1996;22(5):321-324

https://doi.org/10.5271/sjweh.148

Issue date: Oct 1996

Human biomonitoring of trace elements -- continued need for critical evaluation

by Nordberg $\mathrm{G}$

This article in PubMed: www.ncbi.nlm.nih.gov/pubmed/8923603

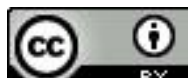




\section{Human biomonitoring of trace elements - continued need for critical evaluation}

Lacking or deficient description of sampling and analytical quality often occurs in scientific publications involving human biomonitoring. In the present issue of this journal, Gerhardsson et al (1) presents an evaluation of publications on reference values for lead in blood. The authors found only 10 out of more than 1000 papers published between 1980 and 1994 which fulfilled a strict set of evaluation criteria.

The expanding number of scientific publications reporting levels of trace elements in human tissues and biological fluids should provide for improved possibilities to define absorbed individual doses in, for example, occupational exposures. In many situations, however, this is difficult because of the lack of access to information about expected "normal" or reference values from occupationally unexposed persons. This problem increases when occupational exposures decrease, as many have done recently, subsequent to the identification of related risks.

One main reason for decreasing concentrations in occupationally exposed persons thus is decreased exposure in the industrial environment. Are reported decreases in "normal" or reference values also explained by decreased exposures?

Yes, but only partly. Reported "background" concentrations of such potentially toxic elements as lead, nickel, chromium, and cadmium in occupationally unexposed persons have decreased substantially, sometimes by a factor as large as 1000 (eg, chromium). The reasons for this decrease in reported concentrations are many, and some have not become obvious until a systematic and critical evaluation of published data has been undertaken.

Several scientists have identified problems in sampling and analysis, but a systematic approach was not taken until an international group of scientists working under the name TRACY (2) was formed on the initiative of the Scientific Committee on Toxicology of Metals within the International Commission on Occupational Health and the Commission on Toxicology within the International Union of Pure and Applied Chemistry. In addition to the TRACY evaluations of published data, the Commission on Toxicology has recently published "Sample Collection Guidelines for Trace Elements in Blood and Urine" (3).

Evaluations by the TRACY Group $(1,2,4-7)$ have shown that there are different reasons for the decreases reported for the various elements. It has, for example, become obvious that most of the decrease in the reported concentrations of nickel and chromium in serum are related to improved control of sampling contamination (stainless needles). For nickel in serum, a measurement increasingly used to evaluate absorbed doses in industrial workers, values reported for occupationally unexposed persons have decreased substantially during the last decade (4), and this decrease has probably been mainly due to improved control over contamination during sampling. However, even for values reported more recently, sufficient control over this error has not always been achieved, and this factor still plays a role in influencing reported values (4). The same situation may be true for chromium (5), and, in addition, several other factors can be expected to influence the values of these two elements. Before analytical and contamination factors are under control, it is not possible to identify other factors, such as dietary intake and internal exposures from prosthetic devices, that are likely to influence the values (5).

As pointed out by Gerhardsson et al in this issue (I), the decreases for lead are only to a limited degree related to contamination problems in sampling, and most of the decrease is actually related to decreased exposure of the general public. This critical evaluation according to the TRACY protocol has 
indicated that, for lead in blood, several factors are important, such as age, gender, ethnicity, food, drinking and smoking habits, hobbies, season and year of sampling, residential area, and geographic location. Since lead in blood is dependent on exposure time and is area specific, it is obvious that it is not possible to present a general overall reference value for lead in the blood of nonoccupationally exposed populations. Instead, it is obvious that the values obtained from occupationally exposed persons must be compared with those of reference groups displaying the same general characteristics as the occupationally exposed group, except for the occupational exposure.

It has been attempted, within the framework of the TRACY criteria, to define more generally applicable reference values. A description of prerequisites and methods, taking cadmium in blood as an example, has been presented by Alessio et al (6). It was obvious however that also for that element all available data could not be accommodated into one common range but that consideration needs to be taken of smoking habits, since they influence blood cadmium values substantially. In addition, specific consideration was necessary for certain areas of the world where dietary cadmium intake is higher than elsewhere. As pointed out in the evaluation of lead (1), geographic variation may be common, and, for most elements, reference values applicable in large geographic areas may not be possible to define.

For cadmium, no important temporal variations could be demonstrated, like those shown for lead. For selenium, however, important annual differences have been shown to exist, depending on fluctuations in dietary intake (7). The evaluation for selenium further demonstrated the usefulness of the TRACY criteria also for an essential element, and it is expected that further evaluations of other essential elements may be forthcoming.

A main conclusion from the TRACY project, so far, is that there are too few publications that contain adequate descriptions of sampling and analysis and therefore only a very small proportion of published data is useful for a discussion of true reference values when strict criteria are applied. This small amount of data makes it easy to select the papers that fulfill the criteria and also to select reliable values, but for several elements the possibilities to identify true factors including variations in background exposure are seriously limited because errors in sampling (including contamination) obscure such variation. It seems obvious that there is still a need for continuous critical evaluation of published data on trace elements in human biomonitoring, and the TRACY project can be expected to produce interesting reports in the future.

It seems peculiar that, in spite of seemingly adequate peer review in reputable journals, so many papers have been published which lack appropriate documentation of quality control. This lack may be due to the editorial policies of some scientific journals according to which authors are discouraged from presenting full population descriptions, sampling details, and analytical performance data in a single manuscript. Editors would be well advised to change such policies and pay more attention to these quality aspects when evaluating and accepting manuscripts for publication.

\section{References}

1. Gerhardsson L, Kazantzis G, Schütz A. Evaluation of selected publications on reference values for lead in blood - The TRACY project. Scand J Work Environ Health 1996;22:325-331.

2. Vesterberg O, Alessio L, Brune D, Gerhardsson L, Herber R, Kazantis G, et al. International project for producing reference values for concentrations of trace elements in human blood and urine - TRACY. Scand J Work Environ Health 1993;19 suppl 1:19-26.

3. Cornelis R, Heinzow B, Herber RFM, Molin Christensen J, Paulsen OM, Sabbioni E, et al. Sample collection guidelines for trace elements in blood and urine. Pure Appl Chem 1995;67:1575-608.

4. Templeton DM, Sunderman Jr SW, Herber RFM. Tentative reference values for nickel concentrations in human serum, plasma, blood, and urine: evaluation according to the TRACY protocol. Sci Total Environ 1994;148:243-51.

5. Brune D, Aitio A, Nordberg G, Vesterberg O, Gerhardsson L. Normal concentrations of chromium in serum and urine - a TRACY project. Scand J Work Environ Health 1993;19 suppl 1:39- 44. 
6. Alessio L, Apostoli P, Braga M, Duca PG, Herber RFM, Nordberg G, et al. Estimation of pooled reference values for cadmium in blood using meta-analysis and TRACY criteria. Sci Total Environ 1994;152:169—77.

7. Alfthan $G$, Neve J. Reference values for serum selenium in various areas - evaluated according to the TRACY protocol. J Trace Elem Med Biol 1996;10:77--87.

Gunnar Nordberg

Department of Occupational and Environmental Medicine

University of Umeå

S-90187 Umeå, Sweden 